\section{Radiochemotherapie oder Operation bei HNSCC?}

\author{
Welche Strategie ist für Patienten mit nicht metastasiertem Platten- \\ epithelkarzinom an Kopf oder Hals (HNSCC) im Stadium III/IV besser: die \\ gleichzeitige Chemo- und Radiotherapie oder Operation mit nachfolgender \\ adjuvanter Bestrahlung? Langzeitergebnisse geben Aufschluss.
}

\begin{abstract}
D andomisiert waren 119 Patienten mit $\{$ neu diagnostiziertem, nicht metastasiertem HNSCC im Stadium III/IV entweder operiert und anschließend bestrahlt worden (RT) oder hatten gleichzeitig eine Chemotherapie mit Cisplatin und 5-Fluorouracil plus RT erhalten. Drei von 75 Patienten, die auf humane Papillomaviren getestet wurden, waren positiv. Wegen schlechter Rekrutierung wurde die Studie vorzeitig gestoppt.

Bis zum letzten Untersuchungszeitpunkt waren 92 Patienten gestorben. Die mediane Beobachtungsdauer betrug 13 Jahre. Eine Analyse der gesamten Kohorte ergab keinen signifikanten Unterschied im Gesamtüberleben und im krankheitsfreien Überleben zwischen
\end{abstract} raum und in der Kieferhöhle beruhte.

Für Patienten mit Tumoren im Mundraum war das Überleben bei einer Operation signifikant besser als bei einer Radiochemotherapie (krankheitsfreie 5-Jahres-Überlebensrate: 68 vs. $12 \%$; $\mathrm{p}=0,038$ ). Bei Patienten mit Tumoren in der Kieferhöhle waren es 71 im Vergleich zu $0 \%(\mathrm{p}=0,05)$.

Fazit: Die gleichzeitige Radiochemotherapie ist eine akzeptable Option für bestimmte Patientengruppen mit Tumoren des Oropharynx, des Pharynx oder des Hypopharynx, mit der Aussicht auf Organerhalt und vielversprechenden Überlebensdaten. Aber diese Option sollte für Patienten mit Tumoren an anderen Lokalisationen nicht empfohlen werden, sofern diese resezierbar sind. Denn die Langzeitergebnisse zeigen einen signifikanten Vorteil für die primäre Operation bei Patienten mit Tumoren in Mundraum oder Kieferhöhle, was sehr für die Operation als Therapie der Wahl bei diesen Patienten spricht.

Judith Neumaier

\section{Familiäres nichtmedulläres Schilddrüsenkarzinom aggressiver als sporadische Form}

\begin{abstract}
Nichtmedulläre Schilddrüsenkarzinome treten überwiegend sporadisch auf, seltener bei positiver Familienanamnese. Dass diese Tumoren aggressiver verlaufen als die sporadischen Formen, belegt eine Metaanalyse.
\end{abstract}

$\mathrm{N}$ ichtmedulläre Schilddrüsenkarzinome (NMTC) entwickeln sich aus follikulären Zellen des Schilddrüsengewe-

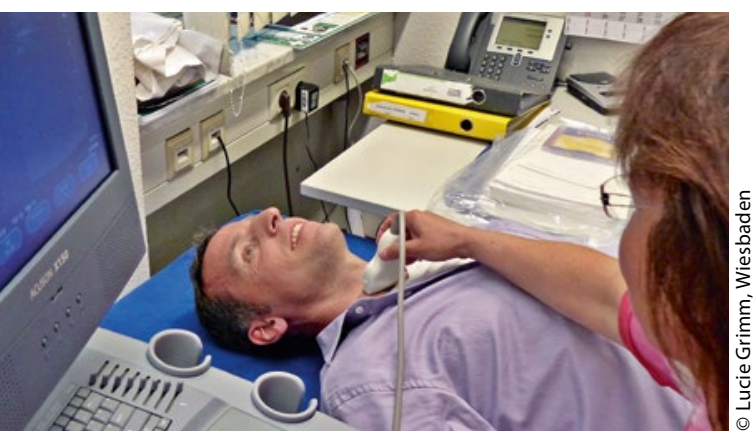

Das familiäre nichtmedulläre Schilddrüsenkarzinom tritt früher auf und ist aggressiver als die sporadische Form. bes und verkörpern $95 \%$ aller TC. Unterschieden werden die vier histologischen Subtypen papilläres, follikuläres, Hürthle-Zell- und anaplastisches TC, die überwiegend sporadisch auftreten. Im letzten Jahrzehnt rückte die familiäre Form des nichtmedullären TC (fNMTC) als offensichtlich klinisch eigenständige Entität in den Vordergrund: Die Patienten hatten mindestens einen ebenfalls betroffenen Verwandten ersten Grades - allerdings ohne weitere gehäuft auftretende potenziell bösartige Syndrome. Die Inzidenz der fNMTC könnte bis zu rund $10 \%$ aller nichtmedullären TC betragen. Der Frage, ob es sich um eine besonders aggressive Form handelt, ging das Team um Xiaofei Wang in einem systematischen Review mit Metaanalyse nach.
Einbezogen in die Analyse waren 12 Originalarbeiten über insgesamt 12.741 Patienten mit familiären und sporadischen NMTC. In den gepoolten Analysen ergaben sich für die familiäre Form ein erhöhtes Rezidivrisiko (Odds Ratio [OR] 1,72) und ein verkürztes krankheitsfreies Überleben (Hazard Ratio [HR] 1,83) jeweils im Vergleich zu den sporadischen Formen. Das fNMTC war darüber hinaus aggressiver, gekennzeichnet durch ein jüngeres Alter bei Diagnosestellung sowie durch ein höheres Risiko für multifokale bzw. bilaterale bzw. extrathyroidale Invasion (OR 1,50 bzw. OR 1,29 bzw. OR 1,20).

Fazit: Das familiär gehäuft auftretende nichtmedulläre TC ist im Vergleich zu den sporadischen Formen aggressiver. Die erhöhte Rezidiv- und Metastasenrate sollte bei den Therapieentscheidungen berücksichtigt werden.

Barbara Kreutzkamp

Wang $X$ et al. Endocrine tumors: familial nonmedullary thyroid carcinoma is a more aggressive disease: a systematic review and meta-analysis. Eur J Endocrinol. 2015;172(6):R253-62. 